\title{
The Gravitational Lens System B1030+074. Discovery and Follow-up.
}

\author{
E. Xanthopoulos, I. W. A. Browne, L. J. King, N. J. Jackson, D. R. \\ Marlow, P. N. Wilkinson \\ University of Manchester, NRAL Jodrell Bank, Macclesfield, Cheshire \\ SK11 9DL, England
}

\section{V. E. Koopmans}

Kapteyn Astronomical Institute, P. O. Box 800, 9700 AV Groningen, The Netherlands

\author{
A. R. Patnaik and R. W. Porcas \\ Max-Planck-Institut für Radioastromomie, Auf dem Hügel 69, D 53121, \\ Bonn, Germany
}

\begin{abstract}
We report the discovery of a new double image gravitational lens system B1030+074 which was found during the Jodrell Bank - VLA Astrometric Survey (JVAS). We have collected extensive radio data on the system using the VLA, MERLIN, the EVN and the VLBA as well as HST WFPC2 and NICMOS observations. The lensed images are separated by 1.56 arcseconds and their flux density ratio at centimetric wavelengths is approximately 14:1 although the ratio is slightly frequency dependent and the images appear to be time variable. The HST pictures show both the lensed images and the lensing galaxy close to the weaker image. The lensing galaxy has substructure which could be either part of the galaxy or a companion object. We have modeled B1030+074 using a Singular Isothermal Ellipsoid that yielded a time delay of $156 / \mathrm{h}_{50}$ days. This lens is likely to be suitable for the measurement of the Hubble constant.
\end{abstract}

\section{Introduction and observations}

The Jodrell-Bank VLA Astrometric Survey (JVAS) is a survey of flat-spectrum radio sources one of whose purposes is to search for gravitational lens systems (Patnaik et al. 1992; Browne et al. 1998, Wilkinson et al. 1998). We report here the discovery of such a double system, B1030+074.

The discovery map of $\mathrm{B} 1030+074$ is presented in Figure 1. It shows two distinct compact components with a separation of 1.56 arcsec, with the fainter image, $\mathrm{B}$, at a $\mathrm{PA}$ of $142^{\circ}$ relative to image A. Table 1 presents all the radio observations. In all the radio maps the two components are unresolved. Only the VLBA $5 \mathrm{GHz}$ data with 3 mas resolution were able to resolve at least one of the components, A, which shows a jet-like extension to the North-East (PA of 65 degrees) and 20 mas in length. 

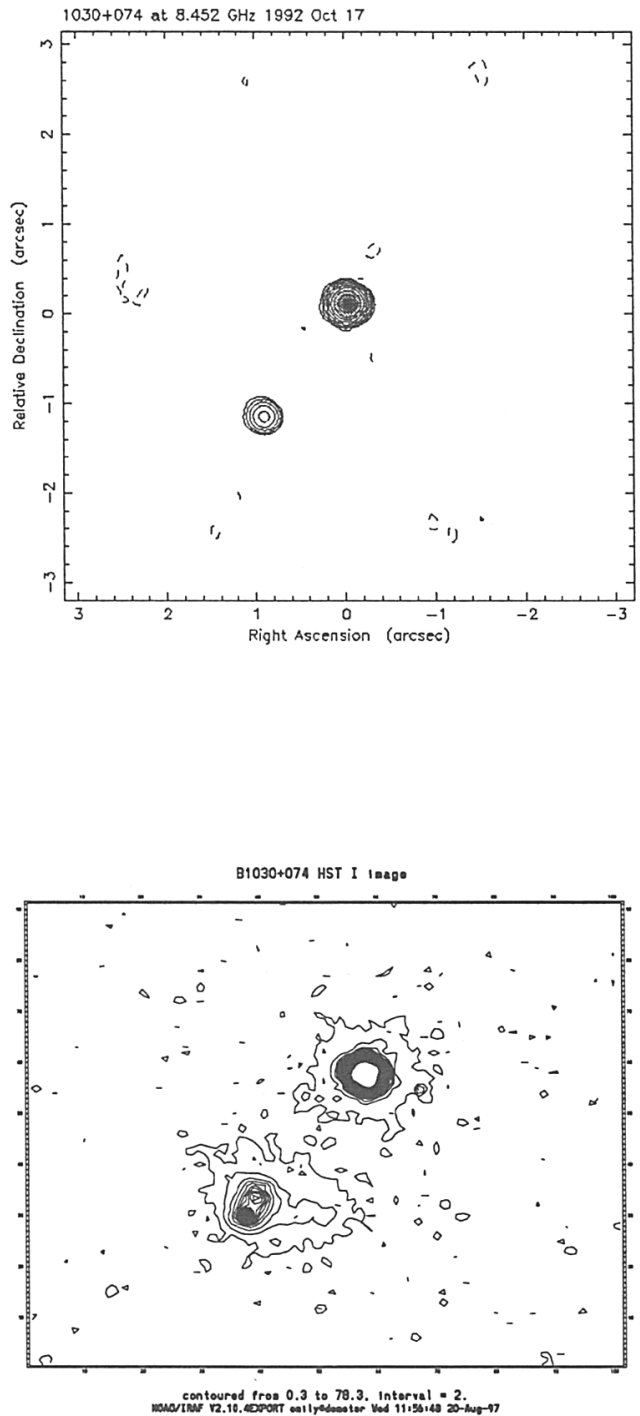

Figure 1. Top: VLA $8.4 \mathrm{GHz} 1992$ discovery map restored with a $200 \times 200$ mas beam. The contours are $0.00037 \mathrm{Jy}$ per beam $\times(-2$, $2,4,8,16,32,64,128,256,512)$, and the peak brightness is $0.189 \mathrm{Jy}$ per beam. Bottom: The HST WFPC2 I image. North is up and East to the left. The lowest contour level is $2 \sigma$ of the sky background value and consecutive contours differ by a factor of 2 in intensity. 


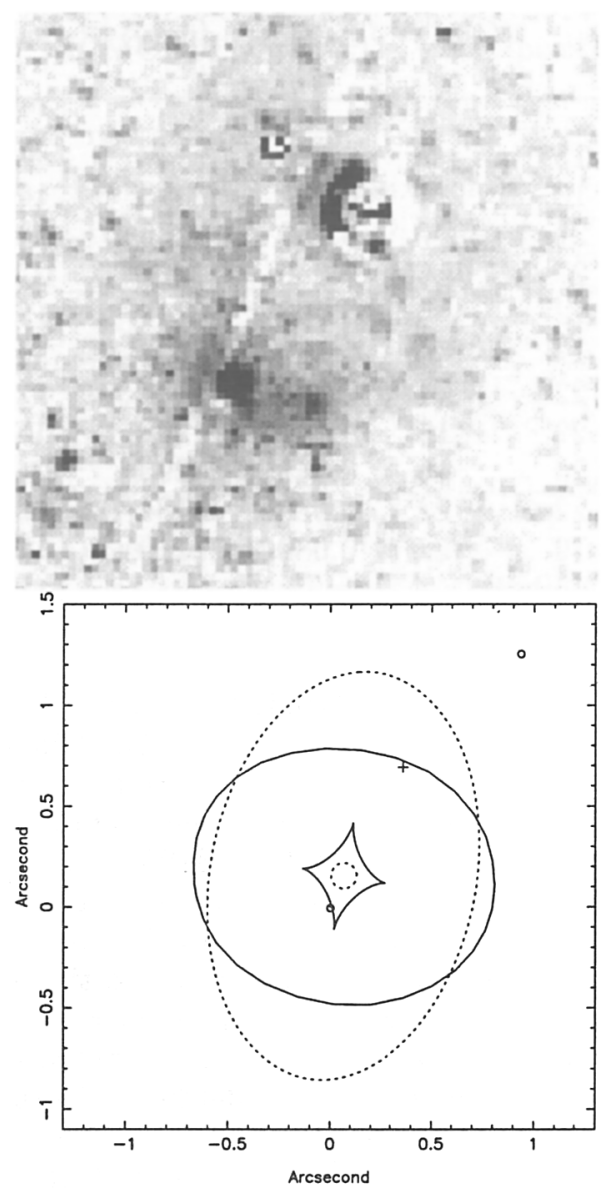

Figure 2. Top: HST NICMOS $\mathrm{H}$ band image after the two point objects $\mathrm{A}$ and $\mathrm{B}$ have been subtracted. The image is 3.69 arcsec on a side. The very bright and close to saturation A image makes a good PSF subtraction difficult. Bottom: The critical (dashed) and caustic (solid) structure of the "best" model of B1030+074. The circles indicate the observed image positions, the cross the inferred source position. 
Table 1. Radio observations of B1030+074. Absolute amplitude errors are estimated to be $5 \%$; the flux density ratios are accurate to $\approx 1 \%$.

$\begin{array}{lrrrrrr}\text { Telesc. } & \text { Obs. date } & \begin{array}{c}\text { Frequ. } \\ (\mathrm{GHz})\end{array} & \begin{array}{c}\text { Resol. } \\ (\mathrm{arcsec})\end{array} & \begin{array}{c}\text { Flux A } \\ (\mathrm{mJy})\end{array} & \begin{array}{c}\text { Flux B } \\ (\mathrm{mJy})\end{array} & \begin{array}{c}\text { Flux } \\ (\mathrm{A} / \mathrm{B})\end{array} \\ & & & & & & \\ \text { EVN } & 19940515 & 1.7 & 0.015 & 147 & 8.1 & 18.1 \\ \text { EVN } & 19941118 & 5 & 0.005 & 173 & 10.9 & 15.9 \\ \text { MERLIN } & 19930927 & 1.7 & 0.150 & 186 & 9.8 & 18.8 \\ \text { MERLIN } & 19961227 & 5 & 0.050 & 326 & 27.3 & 12.0 \\ \text { VLBA } & 19951112 & 5 & 0.003 & 248 & 19.1 & 13.0 \\ \text { VLA } & 19921017 & 8.4 & 0.240 & 202 & 16.0 & 12.6 \\ \text { VLA } & 19940222 & 8.4 & 0.240 & 197 & 12.9 & 15.2 \\ \text { VLA } & 19940222 & 15 & 0.140 & 208 & 14.8 & 14.0 \\ \text { VLA } & 19951220 & 15 & 0.140 & 295 & 24.4 & 12.1 \\ \text { VLA } & 19940222 & 22 & 0.080 & 184 & 15.3 & 12.0 \\ \text { VLA } & 19951219 & 22 & 0.080 & 219 & 12.2 & 18.0\end{array}$

HST images were obtained of $\mathrm{B} 1030+074$ using WFPC2 in two filters F555W and F814W. The contour plot of the I band image is shown in Fig. 1. The contour plots reveal compact optical objects corresponding to both radio components, together with a galaxy between them and very close to the $\mathrm{B}$ component. These data leave no doubt that $\mathrm{B} 1030+074$ is a gravitational lens system. The lensing galaxy appears not to have a simple smooth light distribution nor is it symmetric about the galaxy core. The overall extension of 1.035 arcsec is in a position angle nearly perpendicular to the image separation (6.1 $\mathrm{kpc}$ at the redshift 0.599 of the galaxy assuming $\mathrm{H}_{0}=75 \mathrm{~km} \mathrm{sec}^{-1} \mathrm{Mpc}^{-1}$ and $\mathrm{q}_{0}=0$ ). The redshift of the source is 1.535 . We computed and subtracted scaled PSFs from the direct CCD images. More detail of the lensing galaxy is then revealed. The profile information seems to support the spiral galaxy interpretation for the lensing galaxy since an $\mathrm{r}^{1 / 4}$ law does not fit the surface brightness profile of the galaxy.

NICMOS observations were taken with Camera 1 through the F160W filter. In Fig. 2 we show the NICMOS $1.6 \mu \mathrm{m}$ picture. The peak of the galaxy light lies on the line joining the two images but there is also a secondary emission feature to the SE of the main part of the galaxy. The H-band data indicate that the colour of this feature is similar to that of the rest of the lensing galaxy, possibly suggesting that it is not a spiral arm but either part of the main galaxy or a companion object. Table 2 presents the flux and positions of the two components of the lens system and the lensing galaxy from all three HST band images. The colours of the lensed images are consistent with the lensed object being a quasar or BL Lac object. The optical/infrared flux density ratios of the images are considerably higher than any of those measured in the radio. It is tempting to attribute such differences to extinction but the fact that the H-band ratio is even 
larger than $\mathrm{V}$ and $\mathrm{I}$ ratios argues against this. We suggest that the differences are best explained as arising from microlensing.

Table 2. Optical and F160W image photometry and positions. The galaxy photometry refers to the light within the Einstein radius. Plate scales are $45.5 \mathrm{mas} /$ pixel for the $555-\mathrm{nm}$ and $814-\mathrm{nm}$ images and $43 \mathrm{mas} /$ pixel for NICMOS. Errors in the radio positions are 1 mas or less.

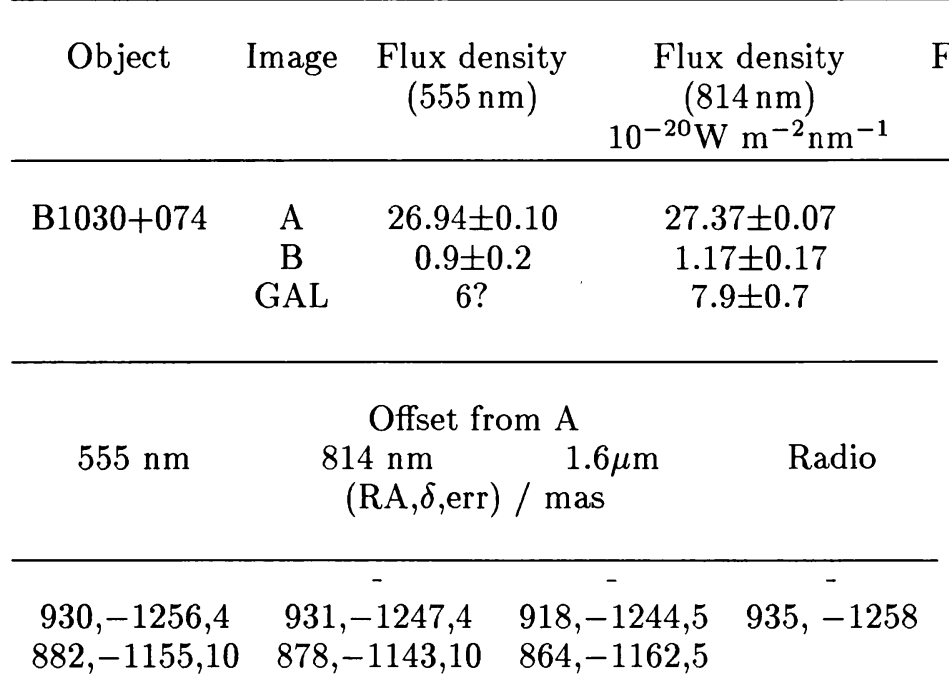

$\mathrm{B} 1030+074$ as a lensed system: There is no doubt that $\mathrm{B} 1030+074$ is a gravitational lens system. We have modeled B1030+074 using a Singular Isothermal Ellipsoid (SIE) mass distribution (Figure 2). To investigate the stability of this model, we performed 10,000 Monte-Carlo simulations, by adding Gaussian distributed errors to the image positions ( 0.3 mas), galaxy position ( 4 mas) and flux density ratio (20\%) (all $1 \sigma$ errors). The predicted time delay is around $156 / h_{50}$ days with an error of only a few percent.

Acknowledgments. This research was supported by European Commission, TMR Programme, Research Network Contract ERBFMRXCT96-0034 "CERES".

\section{References}

Browne, I. W. A., Patnaik, A. R., Wilkinson, P. N., Wrobel, J. M. 1998, MNRAS, 293,257

Patnaik, A. R., Browne, I. W. A., Wilkinson, P. N., Wrobel, J. M. 1992, MNRAS, 254,655

Wilkinson, P. N., et al. 1998, submitted 


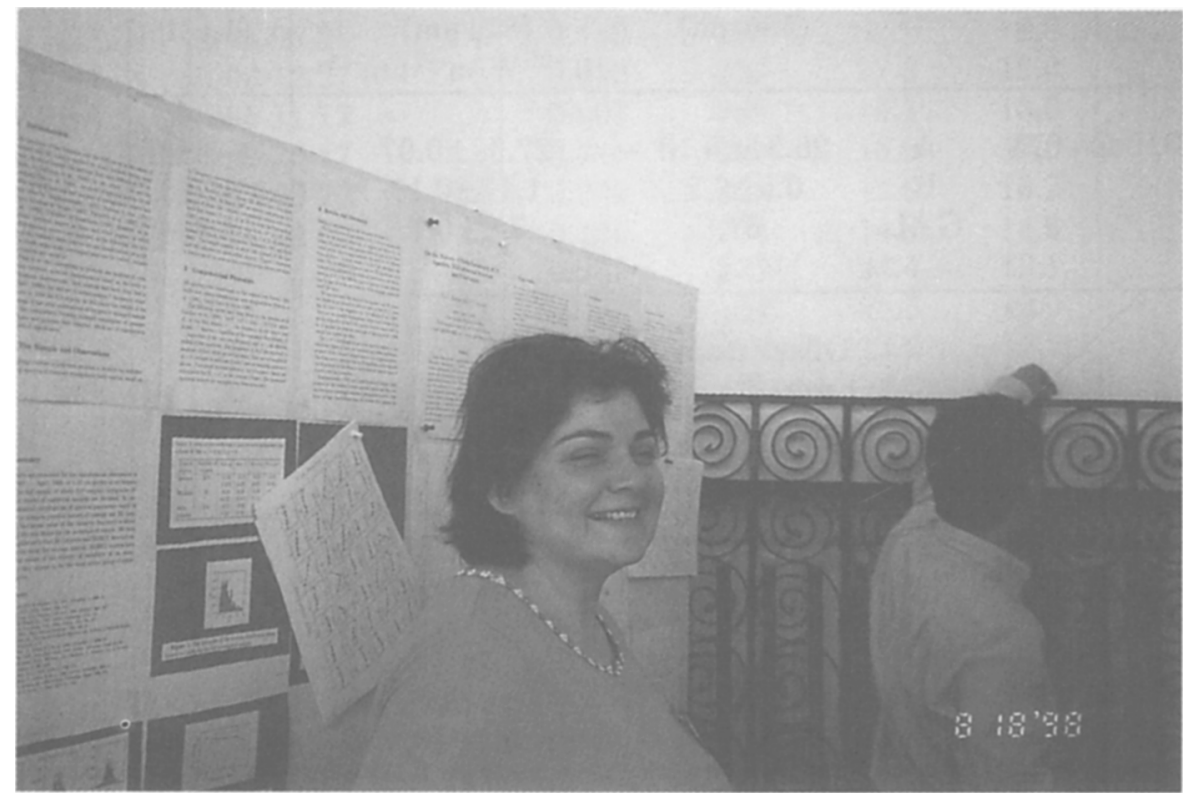

Emily Xanthopoulos 